\title{
Sobolev spaces on multiple cones
}

\author{
P. Auscher \\ Université Paris-Sud \\ UMR du CNRS 8628 \\ F-91405 Orsay Cedex \\ pascal.auscher@math.u-psud.fr \\ N. Badr \\ Institut Camille Jordan \\ Université Claude Bernard Lyon 1 \\ UMR du CNRS 5208 \\ 43 boulevard du 11 novembre 1918 \\ F-69622 Villeurbanne cedex \\ badr@math.univ-lyon1.fr
}

october 28,2008

\begin{abstract}
The purpose of this note is to discuss how various Sobolev spaces defined on multiple cones behave with respect to density of smooth functions, interpolation and extension/restriction to/from $\mathbb{R}^{n}$.

2000 Mathematics Subject Classification. 46B70, 46E35, 42B20.

Key words and phrases. Interpolation; Sobolev spaces; Poincaré inequality; Doubling property; Metric-measure spaces; Calderón-Zygmund decomposition.
\end{abstract}

\section{Introduction}

The theory of Sobolev spaces on domains of the Euclidean spaces is well developed and numerous works and books are available. For multi-connected open sets, there is apparently nothing to say. However, depending on the topology of the boundary, the closure of the space of test functions (ie compactly supported in $\mathbb{R}^{n}$ ) might be a subtle thing. We propose here to investigate the Sobolev spaces on multiple cones with common vertex as unique common point of their boundaries. Surprisingly, we did not find a treatment in the literature.

Our motivation comes from N. Badr's PhD thesis where interpolation results for Sobolev spaces on complete metric measured spaces are proved upon the doubling property and families of Poincaré inequalities. A question remained unsettled, namely whether the result is sharp, that is whether the conclusion 
is best possible given the hypotheses. (Closed) multiple cones are sets where doubling (for Lebesgue measure) holds and $L^{p}$-Poincaré inequalities hold for some but not all $p$, more precisely for $p$ greater than dimension. The study of Sobolev spaces on such sets provides us with the positive answer to our question and, in addition, we complete the interpolation result in this specific situation. As we shall see, these Sobolev spaces can be identified with the closure of test functions in the classical Sobolev space on open multiple cones.

For simplicity, we work on $\Omega$ the Euclidean (double) cone defined by $x_{1}^{2}+$ $\ldots+x_{n-1}^{2}<x_{n}^{2}, n \geq 2$, but all the material extends right away to multiple cones with common vertex point (see Section 7), and the cones need not be of revolution type. The various first order Sobolev spaces on $\Omega$ we consider are: $W_{p}^{1}(\Omega), \widetilde{W}_{p}^{1}(\Omega)$ the closure of smooth compactly supported functions in $\mathbb{R}^{n}$ in $W_{p}^{1}(\Omega)$ and also $H_{p}^{1}(X)$ the Sobolev space arising from geometric measure theory on $X=\bar{\Omega}$.

The question we ask is: how do they behave with respect to density of smooth functions, interpolation and extension/restriction to/from $\mathbb{R}^{n}$ ?

Our results (Sections 2,3,4 and 5) exhibit the specific role of the vertex point. This role translates into a critical exponent (equal to dimension) and the $L^{p}$ Sobolev spaces have different behaviors with respect to the various actions listed above. For example, the usual spaces $W_{p}^{1}(\Omega)$ interpolate for all $p$ in $[1, \infty]$. The space $\widetilde{W}_{p}^{1}(\Omega)$ coincides with $W_{p}^{1}(\Omega)$ if $1 \leq p \leq n$ but is a strict subspace for $n<p$. Hence the way the $\widetilde{W}_{p}^{1}(\Omega)$ interpolate for all $p$ is unclear and we provide an answer, identifying the critical interpolation space at $p=n$, a strict subspace of $\widetilde{W}_{n}^{1}(\Omega)$. The open set $\Omega$ has the extension property for $W_{p}^{1}$ if $p<n$. It cannot be when $p>n$. On the contrary, it is the case if one replaces $W_{p}^{1}(\Omega)$ by $\widetilde{W}_{p}^{1}(\Omega)$. At $p=n$, the extension property is, as we show, not enjoyed by $\widetilde{W}_{n}^{1}(\Omega)$, but by the smaller interpolation space. As for $H_{p}^{1}(X)$ it is easy to show it agrees with $\widetilde{W}_{p}^{1}(\Omega)$ and it turns out that it will be easier to work with the former.

\section{Density}

Let $1 \leq p \leq \infty$ and $O$ an open set of $\mathbb{R}^{n}$. Define $W_{p}^{1}(O)$ as the space of function 1 $f \in L^{p}(O)$ such that

$$
\|f\|_{W_{p}^{1}(O)}=\|f\|_{L^{p}(O)}+\|\nabla f\|_{L^{p}(O)}<\infty .
$$

The gradient is defined in the distributional sense in $O$. For $p<\infty$, denote by $\widetilde{W}_{p}^{1}(O)$ the closure of the space of $C_{0}^{\infty}\left(\mathbb{R}^{n}\right)$ (the subscript 0 means compact support) functions restricted to $O$ in $W_{p}^{1}(O)$. Among classical texts, we quote [1, 13, 14, 15, 17.

If $n<p<\infty$, recall that the Morrey-Sobolev embedding implies that if $f \in W_{p}^{1}(\Omega)$ then $f$ is Hölder continuous on each connected component $\Omega_{ \pm}$of $\Omega$, the half-cones defined by $x \in \Omega$ and $\operatorname{sign}\left(x_{n}\right)= \pm 1$. Hence $f$ has limits in 0 from $\Omega_{+}$and $\Omega_{-}$. These limits, which we call $f\left(0^{+}\right)$and $f\left(0^{-}\right)$, may be different.

\footnotetext{
${ }^{1}$ We consider real functions but everything is valid for complex functions.
} 
Lemma 2.1. Let $1 \leq p<\infty$ and $f \in W_{p}^{1}(\Omega)$. Assume $1 \leq p \leq n$ or $n<p<\infty$ and $f\left(0^{+}\right)=f\left(0^{-}\right)=0$. Then there exists a sequence of $C_{0}^{\infty}\left(\mathbb{R}^{n}\right)$ functions $\left(\varphi_{k}\right)$ with support away from 0 such that $\left\|f-\varphi_{k}\right\|_{W_{p}^{1}(\Omega)}$ tends to 0 .

Proof. We claim that the functions $f \in W_{p}^{1}(\Omega)$ supported away from a ball centered at 0 are dense in $W_{p}^{1}(\Omega)$. Assuming this fact, we argue as follows. Let $f \in W_{p}^{1}(\Omega)$ and we may assume that $f$ is supported away from a ball centered at 0 . One can find a function $h \in W_{p}^{1}\left(\mathbb{R}^{n}\right)$ having the same property for its support such that $f=h$ on $\Omega$. Indeed, extend $\left.f\right|_{\Omega_{+}}$to $h_{+} \in W_{p}^{1}\left(\mathbb{R}^{n}\right)$ in such a way that $h_{+}$vanishes in a neighborhood of 0 in $\mathbb{R}^{n}$ and on $x_{n} \leq 0$. Do the same thing symmetrically for $\left.f\right|_{\Omega_{-}}$to obtain $h_{-}$. Then superpose $h_{+}$and $h_{-}$ to obtain the desired $h$. Next, by convolution and truncation, we approach $h$ in $W_{p}^{1}\left(\mathbb{R}^{n}\right)$ by a function $g \in C_{0}^{\infty}\left(\mathbb{R}^{n}\right)$ supported away from 0 . Since

$$
\|f-g\|_{W_{p}^{1}(\Omega)}=\|h-g\|_{W_{p}^{1}(\Omega)} \leq\|h-g\|_{W_{p}^{1}\left(\mathbb{R}^{n}\right)},
$$

the density result follows.

It remains to prove the above claim. We distinguish between 3 cases:

1) Case $1 \leq p<n$ : Let $f \in W_{p}^{1}(\Omega)$. Write $f=f_{+}+f_{-}$where $f_{+}=$ $\left.f\right|_{\Omega_{+}}$and $f_{-}=\left.f\right|_{\Omega_{-}}$. Since $f_{+}$and $f_{-}$play the same role we can restrict attention to $f_{+}$which we call again $f$. Let $\eta$ be a $C^{\infty}$ function defined on $\mathbb{R}$, $0 \leq \eta \leq 2, \eta=1$ on $[2, \infty[$ and 0 on $]-\infty, 1]$. We take for $x=\left(x_{1}, \ldots, x_{n}\right)$ with $x_{1}, \ldots, x_{n} \in \mathbb{R}, f_{k}(x)=f(x) \eta\left(k x_{n}\right), k \in \mathbb{N}^{*}$. For every $k$, the support of $f_{k}$ does not intersect a ball centered at 0 . Moreover, $f_{k}$ converges to $f$ for the norm $L^{p}\left(\Omega_{+}\right)$. Indeed, $\left|f-f_{k}\right| \leq g_{k}|f|$ where $g_{k}$ is the characteristic function of $B_{k}=\left\{x \in \Omega_{+} ; k x_{n} \leq 2\right\}$ and we apply the Lebesgue dominated convergence theorem. For the partial derivatives we have if $1 \leq i \leq n-1$,

$$
\partial_{x_{i}}\left(f-f_{k}\right)=\partial_{x_{i}} f \eta\left(k x_{n}\right)
$$

and

$$
\partial_{x_{n}}\left(f-f_{k}\right)=\partial_{x_{i}} f \eta\left(k x_{n}\right)+k \eta^{\prime}\left(k x_{n}\right) f .
$$

The $n-1$ first derivatives and the first term in the $n$th derivative are treated as before. For the second one, we have the Sobolev inequality $\|f\|_{L^{p *}}<\infty$ where $p^{*}=\frac{n p}{n-p}$ (in fact we can extend $f$ to $W_{p}^{1}\left(\mathbb{R}^{n}\right)$ and the extension verifies the Sobolev inequality since $p<n$ and $\Omega_{+}$is an extension domain for $W_{p}^{1}$ ). Applying the Hölder inequality one obtains

$$
\begin{aligned}
\left(\int_{B_{k}}\left|k \eta^{\prime}\left(k x_{n}\right) f(x)\right|^{p} d x\right)^{\frac{1}{p}} & \leq\left(\int_{B_{k}}|f|^{p *} d x\right)^{\frac{1}{p^{*}}}\left(\int_{B_{k}} k^{n}\left|\eta^{\prime}\left(k x_{n}\right)\right|^{n} d x\right)^{\frac{1}{n}} \\
& \leq C\left(\int_{B_{k}}|f|^{p *} d x\right)^{\frac{1}{p^{*}}} .
\end{aligned}
$$

Since the right term converges to 0 by the dominated convergence theorem, we conclude that $\nabla\left(f-f_{k}\right)$ converges to 0 in $L^{p}$. 
Case $p=n$ : The density of functions in $W_{n}^{1}\left(\Omega_{ \pm}\right)$supported away from a ball centered at 0 was proved in [7, Lemma 2.4] for the special case $n=2$ (We are thankful to Monique Dauge for indicating this work). The proof applies mutatis mutandis for any $n \geq 2$. Writing $W_{n}^{1}(\Omega)=W_{n}^{1}\left(\Omega_{+}\right) \oplus W_{n}^{1}\left(\Omega_{-}\right)$finishes the proof.

Case $p>n$ : Let $f \in W_{p}^{1}(\Omega)$ such that $f\left(0^{+}\right)=f\left(0^{-}\right)=0$. Write $f=f_{+}+$ $f_{-}$. We can assume $f=f_{+}$. Take $\chi \in C_{0}^{\infty}\left(\mathbb{R}^{n}\right)$ a radial function supported in the unit ball with $\chi=1$ in a neighborhood of 0 . For $\epsilon>0$, define $\chi_{\epsilon}(x)=\chi\left(\frac{x}{\epsilon}\right)$ and take $f_{\epsilon}(x)=f(x)\left(1-\chi_{\epsilon}(x)\right)$. Every $f_{\epsilon}$ is supported away from a ball centered at 0 . Moreover $f_{\epsilon}$ converges to $f$ for the $L^{p}\left(\Omega_{+}\right)$norm by the Lebesgue dominated convergence theorem. For the gradient, we have

$$
\nabla f_{\epsilon}=\nabla f\left(1-\chi_{\epsilon}\right)+f \nabla \chi_{\epsilon} .
$$

The first term also converges to $\nabla f$ in $L^{p}\left(\Omega_{+}\right)$by the Lebesgue dominated convergence theorem. It remains to prove that $\left\|f \nabla \chi_{\epsilon}\right\|_{L^{p}\left(\Omega_{+}\right)}$tends to 0 . By Morrey's theorem and recalling that $f(0)=0$ we have for every $x \in \Omega_{+}|x|<\epsilon$,

$$
\left|\frac{f(x)}{\epsilon}\right| \leq C\left(\frac{|x|}{\epsilon}\right)^{1-n / p}\left(f_{\{|y|<2 \epsilon\} \cap \Omega_{+}}|\nabla f|^{p}(y) d y\right)^{1 / p} .
$$

This implies

$$
\begin{aligned}
\int_{\Omega_{+}} \mid f(x) & \left.\nabla \chi_{\epsilon}(x)\right|^{p} d x \leq \int_{\{|x| \leq \epsilon\} \cap \Omega_{+}}\left|\frac{f(x)}{\epsilon}\right|^{p} d x \\
& \leq \int_{\{|x| \leq \epsilon\} \cap \Omega_{+}}\left(\frac{|x|}{\epsilon}\right)^{p-n} d x \frac{1}{\epsilon^{n}} \int_{\{|y| \leq 2 \epsilon\} \cap \Omega_{+}}|\nabla f|^{p}(y) d y \\
& \leq C \int_{\{|y| \leq 2 \epsilon\} \cap \Omega_{+}}|\nabla f|^{p}(y) d y .
\end{aligned}
$$

We conclude noting that the last integral converges to 0 when $\epsilon \rightarrow 0$ by the dominated convergence theorem.

Corollary 2.2. Let $1 \leq p<\infty$. If $p \leq n, \widetilde{W}_{p}^{1}(\Omega)=W_{p}^{1}(\Omega)$ and if $n<p$, $\widetilde{W}_{p}^{1}(\Omega)=\left\{f \in W_{p}^{1}(\Omega) ; f\left(0^{+}\right)=f\left(0^{-}\right)\right\}$, and hence is of codimension 1 in $W_{p}^{1}(\Omega)$.

Proof. For $1 \leq p \leq n$, the equality follows immediately from Lemma 2.1. Assume now $p>n$. Trivially $\widetilde{W}_{p}^{1}(\Omega) \subset\left\{f \in W_{p}^{1}(\Omega) ; f\left(0^{+}\right)=f\left(0^{-}\right)\right\}$. Conversely let $f \in W_{p}^{1}(\Omega), f\left(0^{+}\right)=f\left(0^{-}\right)$. Then $g=f-f(0) \chi$, with $\chi \in C_{0}^{\infty}\left(\mathbb{R}^{n}\right)$ supported in the unit ball with $\chi \equiv 1$ in a neighborhood of 0 verifies $g\left(0^{+}\right)=$ $g\left(0^{-}\right)=0$. Lemma 2.1 yields $g \in \widetilde{W}_{p}^{1}(\Omega)$ and therefore $f=g+f(0) \chi$.

\section{Real interpolation}

As far as $W_{p}^{1}(\Omega)$ is concerned, we have if $1 \leq p \leq \infty$ that $W_{p}^{1}(\Omega)=W_{p}^{1}\left(\Omega_{+}\right) \oplus$ $W_{p}^{1}\left(\Omega_{-}\right)$using restriction to $\Omega_{ \pm}$and extension by 0 from $\Omega_{ \pm}$to $\Omega$. That is, if $f \in W_{p}^{1}(\Omega)$, we write

$$
f=\mathbf{1}_{\Omega_{+}} f+\mathbf{1}_{\Omega_{-}} f .
$$


Since $\Omega_{ \pm}$is a Lipschitz domain, it is known [8] that the family of Sobolev spaces $\left(W_{p}^{1}\left(\Omega_{ \pm}\right)\right)_{1 \leq p \leq \infty}$ forms a scale of interpolation spaces for the real interpolation method. Hence the same is true for $\left(W_{p}^{1}(\Omega)\right)_{1 \leq p \leq \infty}$.

There is a second chain of spaces appearing in the axiomatic theory of Sobolev spaces on a metric measured space ([9], [10, [12]). Let $X$ be the closure of $\Omega$. Then $X$ equipped with Euclidean distance and Lebesgue measure, which we denote by $\lambda$, is a complete metric measured space. The balls are the restriction to $X$ of Euclidean balls centered in $X$. For $1 \leq p<\infty$, we denote by $H_{p}^{1}(X)$ the completion for the norm $W_{p}^{1}(\Omega)$ of $\operatorname{Lip}_{0}(X)$, the space of Lipschitz functions in $X$ with compact support. For $p=\infty$, we set $H_{\infty}^{1}(X)=\operatorname{Lip}(X) \cap L^{\infty}(X)$. Identifying a Lipschitz function on $\Omega$ with its unique extension to $X, H_{\infty}^{1}(X)=\left\{f \in W_{\infty}^{1}(\Omega) ; f\left(0^{+}\right)=f\left(0^{-}\right)\right\}$.

We recall the definitions of doubling property and Poincaré inequality:

Definition (Doubling property). Let $(E, d, \mu)$ be a metric-measure space. One says that $E$ satisfies the doubling property $(D)$ if there exists a constant $C>0$ such that for all $x \in E, r>0$ we have

$$
\mu(B(x, 2 r)) \leq C \mu(B(x, r)) .
$$

Definition (Poincaré Inequality). A metric-measure space $(E, d, \mu)$ admits a Poincaré inequality $\left(P_{q}\right)$ for some $1 \leq q<\infty$, if there exists a constant $C>0$, such that for every continuous function $u$ and upper gradient $g$ of $u$, and for every ball $B$ of radius $r>0$ the following inequality holds:

$$
\left(f_{B}\left|u-u_{B}\right|^{q} d \mu\right)^{\frac{1}{q}} \leq C r\left(f_{B} g^{q} d \mu\right)^{\frac{1}{q}} .
$$

In the case of $X$, an upper gradient of $u$ is $|\nabla u|$.

The space $(X, d, \lambda)$ has the doubling property and, as shown in [10] p.17, it supports a $p$-Poincaré inequality if and only if $n<p$. It is then a consequence of N. Badr's theorem ([3], Theorem 7.11) that the family $\left(H_{p}^{1}(X)\right)_{n<p \leq \infty}$ is a scale of interpolation spaces for the real interpolation method. Due to some additional specificity of $X$, she was also able to identify $H_{p}^{1}(X)$ as the interpolation space $\left(H_{p_{0}}^{1}(X), H_{p_{1}}^{1}(X)\right)_{\theta, p}$ when $1 \leq p_{0}<p<p_{1} \leq \infty$ and $1 / p=(1-\theta) / p_{0}+\theta / p_{1}$ with the restriction that either $n<p$ or $p_{1}<n$ ([2], [3]).

The missing cases are somehow intriguing and for the sake of curiosity we provide a complete picture in the following result. More interestingly, we provide a proof that covers all cases at once.

Theorem 3.1. If $1 \leq p_{0}<p<p_{1} \leq \infty$ and $1 / p=(1-\theta) / p_{0}+\theta / p_{1}$, then

$$
\left(H_{p_{0}}^{1}(X), H_{p_{1}}^{1}(X)\right)_{\theta, p}= \begin{cases}H_{p}^{1}(X), & \text { if } p \neq n, \\ \widehat{H}_{n}^{1}(X), & \text { if } p=n .\end{cases}
$$

We shall see that $\widehat{H}_{n}^{1}(X)$ is a strict subspace of $H_{n}^{1}(X)$. This implies in particular that Badr's interpolation result is sharp in the class of Sobolev spaces on metric measured spaces: in this example, the infimum of Poincaré exponents is also the smallest exponent $p_{0}$ for which the family $\left(H_{p}^{1}(X)\right)_{p_{0}<p \leq \infty}$ is a scale of interpolation spaces for the real interpolation method. Hence, she could not get a better conclusion in general. 
The space $\widehat{H}_{n}^{1}(X)$ will incorporate a sort of Hardy inequality with respect to the vertex point. To describe it, we need the following definition.

Definition. For a function $f: X \rightarrow \mathbb{R}$, we define its radial part $f_{r}$ and its antiradial part $f_{a}$ as follows: $f_{r}(x)$ is the mean of $f$ on the sphere of radius $|x|$ restricted to $\Omega$ with respect to surface measure and $f_{a}(x)=f(x)-f_{r}(x)$.

The number $f_{r}(x)$ depends only on the distance of $x$ to the origin, hence the terminology radial (even if $\Omega$ is not invariant by rotations). But note that both $f_{r}$ and $f_{a}$ depend on $\Omega$. Note that $f \mapsto f_{r}$ is a contraction on $H_{p}^{1}(X)$. Denote by $r: \mathbb{R}^{n} \rightarrow \mathbb{R}, r(x)=|x|$.

Definition. $\widehat{H}_{n}^{1}(X)=\left\{f \in H_{n}^{1}(X) ; f_{a} / r \in L^{n}(X)\right\}$ with norm

$$
\|f\|_{\widehat{H}_{n}^{1}(X)}=\|f\|_{H_{n}^{1}(X)}+\left\|f_{a} / r\right\|_{L^{n}(X)} .
$$

The following example shows that $\widehat{H}_{n}^{1}(X)$ is a strict subspace of $H_{n}^{1}(X)$. Assume $n=2$ and $\beta>0$, and consider the function $f$ on $X$, supported on $r \leq 1 / 2, C^{\infty}$ away from 0 , which is $\operatorname{sign}\left(x_{2}\right)|\ln r|^{-\beta}$ for $r \leq 1 / 4$. It is easy to check that $f \in H_{2}^{1}(X)$ for all $\beta>0$. Clearly, $f=f_{a}$ and $f / r \in L^{2}(X)$ if and only if $\beta>1 / 2$. Hence for $0<\beta \leq 1 / 2$ we have $f \notin \widehat{H}_{2}^{1}(X)$.

Before we move on, the relation between $H_{p}^{1}(X)$ and $W_{p}^{1}(\Omega)$ is the following.

Lemma 3.2. For $1 \leq p<\infty, H_{p}^{1}(X)=\widetilde{W}_{p}^{1}(\Omega)$ with the same norm.

Proof. It is clear that $\widetilde{W}_{p}^{1}(\Omega) \subset H_{p}^{1}(X) \subset W_{p}^{1}(\Omega)$. Thanks to Corollary 2.2. we have our conclusion if $1 \leq p \leq n$. Assume next $n<p$. Then functions in $\operatorname{Lip}_{0}(X)$ satisfy $f\left(0^{+}\right)=f\left(0^{-}\right)$. Since $f \mapsto f\left(0^{ \pm}\right)$are continuous on $W_{p}^{1}(\Omega)$, this passes to $H_{p}^{1}(X)$. Applying again Corollary 2.2, we deduce that $H_{p}^{1}(X) \subset$ $\widetilde{W}_{p}^{1}(\Omega)$.

To prove our theorem, we first introduce the following spaces.

Definition. For $1 \leq p \leq \infty$, set $\widetilde{H}_{p}^{1}(X)=\left\{f \in H_{p}^{1}(X) ; f / r \in L^{p}(X)\right\}$ with norm

$$
\|f\|_{\widetilde{H}_{p}^{1}(X)}=\|f\|_{H_{p}^{1}(X)}+\|f / r\|_{L^{p}(X)}=\|f\|_{W_{p}^{1}(\Omega)}+\|f / r\|_{L^{p}(\Omega)} .
$$

Lemma 3.3. For $1 \leq p \leq \infty, \widetilde{H}_{p}^{1}(X)$ is a Banach space which can be identified isometrically to $\left\{f \in W_{p}^{1}(\Omega) ; f / r \in L^{p}(\Omega)\right\}$.

Proof. There is nothing to prove if $1 \leq p \leq n$ thanks to Corollary 2.2 and Lemma 3.2. Assume next $n<p<\infty$. Let $f \in \widetilde{H}_{p}^{1}(X)$, then the restriction of $f$ to $\Omega$ belongs to $\left\{f \in W_{p}^{1}(\Omega) ; f / r \in L^{p}(\Omega)\right\}$. Conversely if $f \in W_{p}^{1}(\Omega)$ and $f / r \in L^{p}(\Omega)$, then $f$ has a unique extension to a Hölder continuous function in both $\overline{\Omega_{ \pm}}$. The condition $f / r \in L^{p}(\Omega)$ forces $f\left(0^{+}\right)=f\left(0^{-}\right)=0$. Hence this extension is in $H_{p}^{1}(X)$ and thus in $\widetilde{H}_{p}^{1}(X)$.

The next result is the main step.

Theorem 3.4. The family $\left(\widetilde{H}_{p}^{1}(X)\right)_{1 \leq p \leq \infty}$ is a scale of interpolation spaces for the real interpolation method.

This result is proved in the next section. We continue with 
Proposition 3.5. If $1 \leq p<n, \widetilde{H}_{p}^{1}(X)=H_{p}^{1}(X)$. If $n<p \leq \infty, \widetilde{H}_{p}^{1}(X)=$ $\left\{f \in H_{p}^{1}(X) ; f(0)=0\right\}$ and has codimension 1 in $H_{p}^{1}(X)$.

Before we prove this proposition we need the following lemma (we thank M. Pierre for indicating a simple proof):

Lemma 3.6. Let $1 \leq p \leq \infty$ with $p \neq n$. Then there exists a constant $C=$ $C(p, \Omega)$ such that

$$
\int_{\Omega}\left|\frac{f}{r}\right|^{p} d x \leq C \int_{\Omega}|\nabla f|^{p} d x
$$

for every $f \in H_{p}^{1}(X)$ with, in addition, $f(0)=0$ if $p>n$ (with the usual $L^{\infty}$ norm if $p=\infty$.)

The example above shows that the lemma is false when $p=n$.

Proof. Assume first $1 \leq p<n$. Take $f \in \operatorname{Lip}_{0}(X)$. We have

$$
\begin{aligned}
\int_{\Omega_{+}}\left|\frac{f}{r}\right|^{p} d x & =\int_{\Omega_{+} \cap S_{1}} \int_{0}^{\infty} r^{n-1-p}|f(r, \theta)|^{p} d r d \sigma(\theta) \\
& =\int_{\Omega_{+} \cap S_{1}}\left[\frac{1}{n-p} r^{n-p}|f(r, \theta)|^{p}\right]_{0}^{\infty} d \sigma(\theta) \\
& -\int_{\Omega_{+} \cap S_{1}} \int_{0}^{\infty} \frac{1}{n-p} r^{n-p} p|f|^{p-1} \operatorname{sign} f \frac{\partial f}{\partial r} d r d \sigma(\theta) \\
& =-\frac{p}{n-p} \int_{\Omega_{+} \cap S_{1}} \int_{0}^{\infty}\left|\frac{f}{r}\right|^{p-1} \operatorname{sign} f \frac{\partial f}{\partial r} r^{n-1} d r d \sigma(\theta) \\
& \leq \frac{p}{n-p}\left(\int_{\Omega_{+} \cap S_{1}} \int_{0}^{\infty}\left|\frac{f}{r}\right|^{p} r^{n-1} d r d \sigma(\theta)\right)^{\frac{p-1}{p}} \\
& \left(\int_{\Omega_{+} \cap S_{1}} \int_{0}^{\infty}\left|\frac{\partial f}{\partial r}\right|^{p} r^{n-1} d r d \sigma(\theta)\right)^{\frac{1}{p}} .
\end{aligned}
$$

After simplification, we get $(\underline{3.3})$ on $\Omega_{+}$. We do the same for the integral on $\Omega_{-}$and therefore (3.3) holds for every $f \in \operatorname{Lip}_{0}(X)$. By density, (3.3) holds for every $f \in H_{p}^{1}(X)$.

Assume next $n<p<\infty$. Let $f \in \operatorname{Lip}_{0}(X)$ such that $f(0)=0$. We denote $A=\int_{\Omega_{+} \cap\{|x|>\epsilon\}}\left|\frac{f}{r}\right|^{p} d x$, where $\epsilon>0$. By Morrey's theorem, we have for every $x \in \Omega,|f(x)| \leq C\||\nabla f|\|_{p}|x|^{\alpha}$ with $\alpha=1-n / p$. Repeating the computation 
of (3.3) and since $f$ has a compact support, one obtains

$$
\begin{aligned}
A & =\int_{\Omega_{+} \cap S_{1}} \int_{\epsilon}^{\infty} r^{n-1-p}|f(r, \theta)|^{p} d r d \sigma(\theta) \\
& =\int_{\Omega_{+} \cap S_{1}}\left[\frac{1}{n-p} r^{n-p}|f(r, \theta)|^{p}\right]_{\epsilon}^{\infty} d \sigma(\theta) \\
& -\int_{\Omega_{+} \cap S_{1}} \int_{\epsilon}^{\infty} \frac{1}{n-p} r^{n-p} p|f|^{p-1} \operatorname{sign} f \frac{\partial f}{\partial r} d \sigma(\theta) d r \\
& =\frac{\epsilon^{n-p}}{p-n} \int_{\Omega_{+} \cap S_{1}}|f(\epsilon, \theta)|^{p} d \sigma(\theta)+\frac{p}{p-n} \int_{\Omega_{+} \cap S_{1}} \int_{\epsilon}^{\infty}\left|\frac{f}{r}\right|^{p-1} \operatorname{sign} f \frac{\partial f}{\partial r} r^{n-1} d r d \sigma(\theta) \\
& \leq C^{p}\|\nabla f\|_{p}^{p} \\
& +\frac{p}{p-n}\left(\int_{\Omega_{+} \cap S_{1}} \int_{\epsilon}^{\infty}\left|\frac{f}{r}\right|^{p} r^{n-1} d r d \sigma(\theta)\right)^{\frac{p-1}{p}}\left(\int_{\Omega_{+} \cap S_{1}} \int_{\epsilon}^{\infty}\left|\frac{\partial f}{\partial r}\right|^{p} r^{n-1} d r d \sigma(\theta)\right)^{\frac{1}{p}} .
\end{aligned}
$$

This yields

$$
A \leq C^{p}\||\nabla f|\|_{p}^{p}+\frac{p}{p-n} A^{\frac{p-1}{p}}\||\nabla f|\|_{p} .
$$

Plugging $A^{\frac{p-1}{p}}\||\nabla f|\|_{p} \leq \delta A+\frac{1}{\delta}\||\nabla f|\|_{p}^{p}$ for every $\delta>0$, one obtains

$$
A\left(1-\frac{p}{p-n} \delta\right) \leq\left(C^{p}+\frac{p}{(p-n) \delta}\right)\||\nabla f|\|_{p}^{p} .
$$

Choosing $\delta<\frac{p-n}{p}$, we deduce that

$$
\int_{\Omega_{+} \cap\{|x|>\epsilon\}}\left|\frac{f}{r}\right|^{p} d x \leq C \int_{\Omega_{+}}|\nabla f|^{p} d x .
$$

We then let $\epsilon \rightarrow 0$. We do the same for the integral on $\Omega_{-}$and therefore (3.3) holds for every $f \in \operatorname{Lip}_{0}(X)$ such that $f(0)=0$. By density, (3.3) holds for every $f \in H_{p}^{1}(X)$ such that $f(0)=0$.

When $p=\infty(3.3)$ is a direct consequence of the definition of $H_{\infty}^{1}(X)$ and that $f(0)=0$ with the mean value theorem.

Proof of Proposition 3.5. When $1 \leq p<n$, Lemma 3.6 shows that $H_{p}^{1}(X) \subset$ $\widetilde{H}_{p}^{1}(X)$ and the proposition follows. Now, when $p>n$, Lemma 3.6 yields $\left\{f \in H_{p}^{1}(X) ; f(0)=0\right\} \subset \widetilde{H}_{p}^{1}(X)$. Conversely if $f \in \widetilde{H}_{p}^{1}(X)$, by the continuity of $f$ at 0 and the $L^{p}$ integrability of $f / r$ we easily see that $f(0)=0$.

It remains to prove that $\widetilde{H}_{p}^{1}(X)$ is of codimension 1 in $H_{p}^{1}(X)$. This follows by writing $f \in H_{p}^{1}(X)$ as $f=f-f(0) \chi+f(0) \chi$, where $\chi \in C_{0}^{\infty}\left(\mathbb{R}^{n}\right)$, supp $\chi \subset$ $B(0,1)$ and $\chi=1$ in a neighborhood of 0 , and using the above characterization of $\widetilde{H}_{p}^{1}(X)$.

Although this is a simple description of $\widetilde{H}_{p}^{1}(X)$, the jump at $p=n$ does not allow us to use this result to conclude for Theorem 3.1. We need to further analyze the radial and antiradial parts of a function.

Lemma 3.7. Let $1 \leq p \leq \infty$. 
1. For a function $f$ depending only on the distance to the origin, $f \in H_{p}^{1}(X) \Longleftrightarrow$ $f \in W_{p}^{1}\left(\mathbb{R}^{n}\right)$ with same norm up to a constant.

2. Assume $p \neq n$. For a function $f: X \rightarrow \mathbb{R}$ and $f_{a}=f-f_{r}$, we have $f_{a} \in \widetilde{H}_{p}^{1}(X) \Longleftrightarrow f_{a} \in H_{p}^{1}(X)$ with comparable norms.

Proof. The first item is trivial. The constant is the ratio of the surface measure of $\Omega$ inside the unit sphere divided by the surface measure of the unit sphere.

As for the second item, it follows from the previous proposition directly if $p<n$ and by observing that $f_{a}(0)=0$ if $p>n$.

We can now complete the proof of Theorem 3.1.

Proof. Let us examine the case where neither $p_{0}, p_{1}$ is $n$. By the reiteration theorem, this reduces further to $p_{0}=1, p_{1}=\infty$. Set $F_{p}=\left(H_{1}^{1}(X), H_{\infty}^{1}(X)\right)_{\theta, p}$ with $\theta=1-1 / p$.

Let $f \in F_{p}$. Since $f \mapsto f_{r}$ is contracting on $H_{q}^{1}(X)$ for all $1 \leq q \leq \infty$ and using Lemma 3.7, one has that

$$
K\left(f_{r}, t, W_{1}^{1}\left(\mathbb{R}^{n}\right), W_{\infty}^{1}\left(\mathbb{R}^{n}\right)\right) \leq C K\left(f, t, H_{1}^{1}(X), H_{\infty}^{1}(X)\right) .
$$

$K$ is the $K$-functional of interpolation defined as in [4, [5]. Hence $f_{r} \in W_{p}^{1}\left(\mathbb{R}^{n}\right)$ by classical interpolation for the $W_{p}^{1}\left(\mathbb{R}^{n}\right)$. Thus $f_{r} \in H_{p}^{1}(X)$ by Lemma 3.7. We also have by Lemma 3.7 again,

$$
K\left(f_{a}, t, \widetilde{H}_{1}^{1}(X), \widetilde{H}_{\infty}^{1}(X)\right) \leq C K\left(f, t, H_{1}^{1}(X), H_{\infty}^{1}(X)\right) .
$$

Theorem 3.4 shows then that $f_{a} \in \widetilde{H}_{p}^{1}(X)$. We conclude that $f \in H_{p}^{1}(X)$ if $p \neq n$ and $f \in \widehat{H}_{n}^{1}(X)$ if $p=n$.

Reciprocally, let $f \in H_{p}^{1}(X)$ if $p \neq n$ and $f \in \widehat{H}_{n}^{1}(X)$ if $p=n$. By Lemma 3.7. whatever $p$ is, we have that $f_{r} \in W_{p}^{1}\left(\mathbb{R}^{n}\right)$ and $f_{a} \in \widetilde{H}_{p}^{1}(X)$. By Theorem 3.4. $f_{a} \in\left(\widetilde{H}_{1}^{1}(X), \widetilde{H}_{\infty}^{1}(X)\right)_{\theta, p}$ with $\theta=1-1 / p$. Hence $f_{a} \in F_{p}$. For the radial part, for each $t>0$, one can find a decomposition $f_{r}=g_{t}+h_{t}$ almost minimizing for $K\left(f_{r}, t, W_{1}^{1}\left(\mathbb{R}^{n}\right), W_{\infty}^{1}\left(\mathbb{R}^{n}\right)\right)$ and one can assume both $g_{t}$ and $h_{t}$ are radial. Thus Lemma 3.7 implies that $g_{t} \in H_{1}^{1}(X)$ and $h_{t} \in H_{\infty}^{1}(X)$, hence $f_{r} \in F_{p}$.

It remains to study the case where $p_{0}$ or $p_{1}$ is equal to $n$. Let us consider the case $p_{1}=n$ as the other one is similar. It is also enough to look at the result when $p_{0}=1$. As we know all interpolation spaces between $H_{1}^{1}(X)$ and $H_{\infty}^{1}(X)$, by the reiteration theorem, if $1<p<n$ and $\frac{1}{p}=1-\theta+\frac{\theta}{n}$ we have $\left(H_{1}^{1}(X), \widehat{H}_{n}^{1}(X)\right)_{\theta, p}=H_{p}^{1}(X)$. Hence, we have

$$
H_{p}^{1}(X)=\left(H_{1}^{1}(X), \widehat{H}_{n}^{1}(X)\right)_{\theta, p} \subset\left(H_{1}^{1}(X), H_{n}^{1}(X)\right)_{\theta, p} \subset H_{p}^{1}(X) .
$$

The last inclusion is the easy part of the interpolation. This concludes the proof.

Remark. The inclusion $\widehat{H}_{n}^{1}(X) \subset H_{n}^{1}(X)$ is dense. This is due to the fact that as $H_{n}^{1}(X)=W_{n}^{1}(\Omega)$, the space of restrictions to $X$ of smooth functions on $\mathbb{R}^{n}$ with compact support in $\mathbb{R}^{n} \backslash\{0\}$, a subspace of $\widehat{H}_{n}^{1}(X)$, is dense in $H_{n}^{1}(X)$. 
Remark. As $X$ is symmetric with respect to $S: x \mapsto-x$, we can define $\widehat{H}_{n}^{1}(X)$ differently by doing an analysis with even and odd parts. Define the even and odd parts $f_{e}$ and $f_{o}$ of a function $f: X \rightarrow \mathbb{R}$ as $f_{e}=\frac{1}{2}(f+f \circ S)$ and $f_{o}=\frac{1}{2}(f-f \circ S)$. We have that $\widehat{H}_{n}^{1}(X)=\left\{f \in H_{n}^{1}(X) ; f_{o} / r \in L^{n}(X)\right\}$. Let $f \in H_{n}^{1}(X)$. Write $f_{o}=\left(f_{r}\right)_{o}+\left(f_{a}\right)_{o}$ and easily $\left(f_{r}\right)_{o}=0$. Hence

$$
f_{a} \in \widetilde{H}_{n}^{1}(X) \Longrightarrow\left(f_{a}\right)_{o} \in \widetilde{H}_{n}^{1}(X) \Longrightarrow f_{o} \in \widetilde{H}_{n}^{1}(X) .
$$

Next, write $f_{a}=\left(f_{e}\right)_{a}+\left(f_{o}\right)_{a}$. We claim that $\left(f_{e}\right)_{a} / r \in L^{n}(X)$. Hence,

$$
f_{o} \in \widetilde{H}_{n}^{1}(X) \Longrightarrow\left(f_{o}\right)_{a} \in \widetilde{H}_{n}^{1}(X) \Longrightarrow f_{a} \in \widetilde{H}_{n}^{1}(X) .
$$

To see the claim, we observe that the evenness of $f_{e}$ implies that $\left(f_{e}\right)_{r}(x)$ can be calculated by replacing the mean of $f_{e}$ on $\Omega \cap S_{|x|}$ by the one on $\Omega_{+} \cap S_{|x|}$. This spherical cap is connected and satisfies Poincaré inequalities with respect to surface measure on $S_{|x|}$, so that we obtain,

$$
\int_{\Omega_{+} \cap S_{|x|}}\left|\left(f_{e}\right)_{a}\right|^{n} d \sigma(\theta) \leq C\left(n, \Omega_{+}\right)|x|^{n} \int_{\Omega_{+} \cap S_{|x|}}\left|\nabla_{\theta}\left(f_{e}\right)_{a}\right|^{n} d \sigma(\theta)
$$

were $\nabla_{\theta}$ is the tangential gradient and notice that $\left|\nabla_{\theta}\left(f_{e}\right)_{a}\right| \leq\left|\nabla\left(f_{e}\right)_{a}\right|$ on $\Omega_{+} \cap S_{|x|}$. So multiplying by $r^{-n}=|x|^{-n}$ and integrating with respect to $d r$ we obtain

$$
\int_{\Omega_{+}} \frac{\left|\left(f_{e}\right)_{a}\right|^{n}}{r^{n}} d x \leq C\left(n, \Omega_{+}\right) \int_{\Omega_{+}}\left|\nabla\left(f_{e}\right)_{a}\right|^{n} d x
$$

This inequality is valid with $\mathrm{n}$ replaced by any $1 \leq p \leq \infty$.

\section{Proof of Theorem 3.4}

For the proof of Theorem 3.4, we need a Calderón-Zygmund decomposition as in 3 . We incorporate here a further control to take care of the vertex point.

Let $1<p<\infty$ and $f \in \widetilde{H}_{p}^{1}(X)$. Identifying $f$ to its restriction to $\Omega$, write $f=\left.f\right|_{\Omega_{+}}+\left.f\right|_{\Omega_{-}}=f_{+}+f_{-}$. We establish the following Calderón-Zygmund decomposition for $f_{+}$and the same decomposition holds for $f_{-}$.

Proposition 4.1 (Calderón-Zygmund lemma). Let $\alpha>0$. Then one can find a collection of balls $\left(B_{i+}\right)_{i}$ of $\Omega_{+}$, functions $b_{i+}$ and a Lipschitz function $g_{+}$such that the following properties hold:

$$
\begin{gathered}
f_{+}=g_{+}+\sum_{i} b_{i+} \quad \text { on } \Omega_{+} \\
\left|g_{+}(x)\right|+\frac{\left|g_{+}(x)\right|}{|x|}+\left|\nabla g_{+}(x)\right| \leq C \alpha \quad \lambda-\text { a.e } x \in \Omega_{+} \\
\operatorname{supp} b_{i+} \subset B_{i+}, f_{B_{i+}}\left(\left|b_{i+}\right|+\frac{\left|b_{i+}\right|}{|x|}+\left|\nabla b_{i+}\right|\right) d x \leq C \alpha \\
\sum_{i} \lambda\left(B_{i_{+}}\right) \leq C \alpha^{-p} \int_{\Omega_{+}}\left(\left|f_{+}\right|+\frac{\left|f_{+}\right|}{|x|}+\left|\nabla f_{+}\right|\right)^{p} d x
\end{gathered}
$$




$$
\sum_{i} \chi_{B_{i+}} \leq N
$$

The constants $C$ and $N$ only depend on $p$ and on the constants in $(D)$ and $\left(P_{1}\right)$ in $\Omega_{+}$.

A ball of $\Omega_{+}$is the restriction to $\Omega_{+}$of an open ball of $\mathbb{R}^{n}$ having center in $\Omega_{+}$.

Proof. To simplify the exposition, we omit the index + keeping it only for $\Omega_{+}$. For $x \in \mathbb{R}^{n}$, denote $r(x)=|x|$. Consider

$$
U=\left\{x \in \Omega_{+}: \mathcal{M}_{\Omega_{+}}\left(|f|+\frac{|f|}{r}+|\nabla f|\right)(x)>\alpha\right\}
$$

with

$$
\mathcal{M}_{\Omega_{+}} f(x)=\sup _{B: x \in B} \frac{1}{\lambda(B)} \int_{B}|f| d x
$$

where $B$ ranges over all balls of $\Omega_{+}$. Recall that $\mathcal{M}_{\Omega_{+}}$is of weak type $(1,1)$ and bounded on $L^{p}\left(\Omega_{+}, \lambda\right), 1<p \leq \infty$. If $U=\emptyset$, then set

$$
g=f, \quad b_{i}=0 \text { for all } i
$$

so that (4.2) is satisfied according to the Lebesgue differentiation theorem. Otherwise the maximal theorem gives us

$$
\begin{aligned}
\lambda(U) & \leq C \alpha^{-p} \int_{\Omega_{+}}\left(|f|+\frac{|f|}{r}+|\nabla f|\right)^{p} d x \\
& <+\infty .
\end{aligned}
$$

In particular $U \neq \Omega_{+}$as $\lambda\left(\Omega_{+}\right)=+\infty$. Let $F$ be the complement of $U$ in $\Omega_{+}$. Since $U$ is an open set distinct of $\Omega_{+}$, we use a Whitney decomposition of $U([6])$ : one can find pairwise disjoint balls $\underline{B_{i}}$ of $\Omega_{+}$and two constants $C_{2}>C_{1}>1$, such that

1. $U=\cup_{i} B_{i}$ with $B_{i}=C_{1} \underline{B_{i}}$ and the balls $B_{i}$ have the bounded overlap property;

2. $r_{i}=r\left(B_{i}\right)=\frac{1}{2} d\left(x_{i}, F\right)$ and $x_{i}$ is the center of $B_{i}$;

3. each ball $\overline{B_{i}}=C_{2} \underline{B_{i}}$ intersects $F\left(C_{2}=4 C_{1}\right.$ works $)$.

Recall that the above balls are balls of $\Omega_{+}$, that is $B_{i}=B\left(x_{i}, r_{i} / C_{1}\right) \cap \Omega_{+}$, $B_{i}=B\left(x_{i}, r_{i}\right) \cap \Omega_{+}, \overline{B_{i}}=B\left(x_{i}, r_{i} C_{2}\right) \cap \Omega_{+}$and $x_{i} \in \overline{\Omega_{+}}$where $B(x, r)$ denotes an Euclidean open ball in $\mathbb{R}^{n}$.

Note that $r_{i} \leq r$ in $B_{i}$. For $x \in U$, denote $I_{x}=\left\{i: x \in B_{i}\right\}$. By the bounded overlap property of the balls $B_{i}$, we have that $\sharp I_{x} \leq N$. Fixing $j \in I_{x}$ and using the properties of the $B_{i}$ 's, we easily see that $\frac{1}{3} r_{i} \leq r_{j} \leq 3 r_{i}$ for all $i \in I_{x}$. In particular, $B_{i} \subset 7 B_{j}$ for all $i \in I_{x}$. Condition (4.5) is nothing but the bounded overlap property of the $B_{i}$ 's and (4.4) follows from (4.5) and (4.6). The doubling property of $\Omega_{+}$and the fact that $\overline{B_{i}} \cap F \neq \emptyset$ yield

$$
\int_{B_{i}}\left(|f|+\frac{|f|}{r}+|\nabla f|\right) d x \leq \int_{\overline{B_{i}}}\left(|f|+\frac{|f|}{r}+|\nabla f|\right) d x \leq \alpha \lambda\left(\overline{B_{i}}\right) \leq C \alpha \lambda\left(B_{i}\right) .
$$


Let us now define the functions $b_{i}$. Let $\left(\chi_{i}\right)_{i}$ be a partition of unity of $U$ subordinated to the covering $\left(\underline{B_{i}}\right)$, such that for all $i, \chi_{i}$ is a Lipschitz function supported in $B_{i}$ with $\left\|\left|\nabla \chi_{i}\right|\right\|_{\infty} \leq \frac{C}{r_{i}}$. To this end it is enough to choose $\chi_{i}(x)=\psi\left(\frac{C_{1} d\left(x_{i}, x\right)}{r_{i}}\right)\left(\sum_{k} \psi\left(\frac{C_{1} d\left(x_{k}, x\right)}{r_{k}}\right)\right)^{-1}$, where $\psi$ is a smooth function, $\psi=1$ on $[0,1], \psi=0$ on $\left[\frac{1+C_{1}}{2},+\infty\left[\right.\right.$ and $0 \leq \psi \leq 1$. We set $b_{i}=\left(f-f_{B_{i}}\right) \chi_{i}$. It is clear that $\operatorname{supp} b_{i} \subset B_{i}$. Let us estimate $\int_{B_{i}}\left|b_{i}\right| d x, \int_{B_{i}} \frac{\left|b_{i}\right|}{r_{i}} d x$ and $\int_{B_{i}}\left|\nabla b_{i}\right| d x$. We have

$$
\begin{aligned}
\int_{B_{i}}\left|b_{i}\right| d x & =\int_{B_{i}}\left|\left(f-f_{B_{i}}\right) \chi_{i}\right| d x \\
& \leq C\left(\int_{B_{i}}|f| d x+\int_{B_{i}}\left|f_{B_{i}}\right| d x\right) \\
& \leq C \int_{B_{i}}|f| d x \\
& \leq C \alpha \lambda\left(B_{i}\right) .
\end{aligned}
$$

We applied Jensen's inequality in the second estimate, and (4.7) in the last one. For $\frac{\left|b_{i}\right|}{r}$ we have

$$
\begin{aligned}
\int_{B_{i}} \frac{\left|b_{i}\right|}{r} d x & =\int_{B_{i}} \frac{\left|\left(f-f_{B_{i}}\right) \chi_{i}\right|}{r} d x \\
& \leq \int_{B_{i}} \frac{\left|f-f_{B_{i}}\right|}{r_{i}} d x \\
& \leq C \int_{B_{i}}|\nabla f| d x \\
& \leq C \alpha \lambda\left(B_{i}\right) .
\end{aligned}
$$

Since $\nabla\left(\left(f-f_{B_{i}}\right) \chi_{i}\right)=\chi_{i} \nabla f+\left(f-f_{B_{i}}\right) \nabla \chi_{i}$, the Poincaré inequality $\left(P_{1}\right)$ on $\Omega_{+}$and (4.7) yield

$$
\begin{aligned}
\int_{B_{i}}\left|\nabla b_{i}\right| d x & \leq C\left(\int_{B_{i}}\left|\chi_{i} \nabla f\right| d x+\int_{B_{i}}\left|f-f_{B_{i}}\right|\left|\nabla \chi_{i}\right| d x\right) \\
& \leq C \alpha \lambda\left(B_{i}\right)+C \frac{C}{r_{i}} r_{i} \int_{B_{i}}|\nabla f| d x \\
& \leq C \alpha \lambda\left(B_{i}\right) .
\end{aligned}
$$

Therefore (4.3) is proved. By similar arguments, $\int \frac{\left|b_{i}\right|}{r_{i}} d x \leq C \alpha \lambda\left(B_{i}\right)$.

Set $g=f-\sum_{i} b_{i}$. Since the sum is locally finite on $U, g$ is defined almost everywhere on $\Omega$ and $g=f$ on $F$. Observe that $g$ is a locally integrable function on $\Omega$. Indeed, let $\varphi \in L^{\infty}$ with compact support. Since $d(x, F) \geq r_{i}$ for $x \in \operatorname{supp} b_{i}$ and $\sum \lambda\left(B_{i}\right) \leq C \lambda(U)$ by using doubling and the disjointness 
of the balls $\underline{B_{i}}$, we obtain

$$
\begin{aligned}
\int \sum_{i}\left|b_{i}\right||\varphi| d x & \leq\left(\int \sum_{i} \frac{\left|b_{i}\right|}{r_{i}} d x\right) \sup _{x \in \Omega_{+}}(d(x, F)|\varphi(x)|) \\
& \leq C \alpha \lambda(U) \sup _{x \in \Omega_{+}}(d(x, F)|\varphi(x)|) .
\end{aligned}
$$

Since $f \in L_{l o c}^{1}$, we deduce that $g \in L_{l o c}^{1}$. It remains to prove (4.2). In the sense of distributions, we have

$$
\begin{aligned}
\nabla g & =\nabla f-\sum_{i} \nabla b_{i} \\
& =\nabla f-\left(\sum_{i} \chi_{i}\right) \nabla f-\sum_{i}\left(f-f_{B_{i}}\right) \nabla \chi_{i} \\
& =\mathbb{1}_{F}(\nabla f)-\sum_{i}\left(f-f_{B_{i}}\right) \nabla \chi_{i} .
\end{aligned}
$$

From the definition of $F$ and the Lebesgue differentiation theorem, we have that $\mathbb{1}_{F}\left(|f|+\frac{|f|}{r}+|\nabla f|\right) \leq \alpha \lambda$-a.e.. We claim that a similar estimate holds for $h=\sum_{i}\left(f-f_{B_{i}}\right) \nabla \chi_{i}$, i.e. $|h(x)| \leq C \alpha$ for all $x \in \Omega_{+}$. For this, note first that $h$ vanishes on $F$. Then fix $x \in U$. Observe that $\sum_{i} \nabla \chi_{i}(x)=0$, and by the definition of $I_{x}$, the sum reduces $i \in I_{x}$. Hence, we have for all $j \in I_{x}$,

$$
\sum_{i}\left(f(x)-f_{B_{i}}\right) \nabla \chi_{i}(x)=\sum_{i \in I_{x}}\left(f(x)-f_{B_{i}}\right) \nabla \chi_{i}(x)=\sum_{i \in I_{x}}\left(f_{B_{j}}-f_{B_{i}}\right) \nabla \chi_{i}(x) .
$$

We claim that $\left|f_{B_{j}}-f_{B_{i}}\right| \leq C r_{j} \alpha$ with $C$ independent of $i, j \in I_{x}$ and $x \in U$. Indeed, we use that $B_{i}$ and $B_{j}$ are contained in $7 B_{j}$, Poincaré inequality $\left(P_{1}\right)$, the comparability of $r_{i}$ and $r_{j}$, and (4.7). Since $I_{x}$ has cardinal bounded by $N$, we are done. From these estimates we deduce that $|\nabla g(x)| \leq C \alpha \lambda-$ a.e..

Let us now estimate $\|g\|_{\infty}$. We have $g=f \mathbb{1}_{F}+\sum_{i} f_{B_{i}} \chi_{i}$. Since $|f| \mathbb{1}_{F} \leq \alpha$, it remains to estimate $\left\|\sum_{i} f_{B_{i}} \chi_{i}\right\|_{\infty}$. Note that since $\overline{B_{i}} \cap F \neq \emptyset$

$$
\left|f_{B_{i}}\right| \leq C f_{\overline{B_{i}}}|f| d x \leq C \alpha
$$

Since $\sum_{i} \chi_{i}=1$ on $U$, inequality (4.8) yields for $x \in U$,

$$
|g(x)|=\left|\sum_{i} f_{B_{i}} \chi_{i}(x)\right| \leq C \alpha \sum_{i} \chi_{i}(x)=C \alpha
$$

We conclude that $\|g\|_{\infty} \leq C \alpha \quad \lambda-$ a.e..

We still need to estimate $\left\|\frac{g}{r}\right\|_{\infty}$. On $F,\left|\frac{g}{r}\right| \leq C \alpha$. Take now $x \in U$. For all $i \in I_{x}$,

$$
\frac{\left|f_{B_{i}}\right|}{|x|} \leq C f_{B_{i}} \frac{|f(y)|}{|y|} d y \leq C \alpha \text {. }
$$

\footnotetext{
${ }^{2}$ Note that since $b \in L^{1}$ in our case, we can say directly that $g \in L_{l o c}^{1}$. However, this way of doing applies to the homogeneous case presented in Section 6.
} 
Then

$$
\frac{|g(x)|}{|x|} \leq \sum_{i \in I_{x}} \frac{\left|f_{B_{i}}\right|}{|x|} \chi_{i}(x) \leq C \alpha \sum_{i \in I_{x}}\left|\chi_{i}(x)\right| \leq C \alpha .
$$

We are now able to characterize the $K$-functional of interpolation between $\widetilde{H}_{1}^{1}(X)$ and $\widetilde{H}_{\infty}^{1}(X)$.

Theorem 4.2. We have that

$$
K\left(f, t, \widetilde{H}_{1}^{1}, \widetilde{H}_{\infty}^{1}\right) \sim t\left(f^{* *}(t)+\left(\frac{|f|}{r}\right)^{* *}(t)+|\nabla f|^{* *}(t)\right)
$$

for every $f \in \widetilde{H}_{1}^{1}(X)+\widetilde{H}_{\infty}^{1}(X)$ and $t>0$. The implicit constants are independent of $f$ and $t$.

Proof. The lower bound follows from the fact that $K\left(g, t, L^{1}, L^{\infty}\right) \sim t g^{* *}(t)$ for $g \in L^{1}+L^{\infty}$. Now for the upper bound, consider first the case when $f \in \widetilde{H}_{p}^{1}(X)$. Identifying $f$ to its restriction to $\Omega$, write $f=f_{+}+f_{-}$and take the above Calderón-Zygmund decomposition for each $f_{+}$and $f_{-}$for $\alpha>0$ to be chosen. We obtain open subsets $U_{ \pm}$and functions $g_{ \pm}, b_{ \pm}$. We assume that $U_{ \pm}$are nonempty; the easy modifications otherwise are left to the reader.

Here is the point of working with the $\widetilde{H}$ spaces instead of the $H$ spaces. As $g_{+}\left(0^{+}\right)=g_{-}\left(0^{-}\right)=0$, if we define $g=g_{+}$on $\Omega_{+}$and $g_{-}$on $\Omega_{-}$, then $g$ can be extended to a Lipschitz function on $X=\bar{\Omega}$ with $\left\|\frac{g}{r}\right\|_{\infty} \leq C \alpha$. Hence $g \in \widetilde{H}_{\infty}^{1}(X)$ with norm controlled by $C \alpha$.

Therefore we can write $f \in \widetilde{H}_{p}^{1}(X)$ as $f=g+b$ with $b \in W_{1}^{1}(\Omega)=\widetilde{H}_{1}^{1}(X)$ and $g \in \widetilde{H}_{\infty}^{1}(X)$. We have $\|g\|_{\widetilde{H}_{\infty}^{1}(X)} \leq C \alpha$ and $\|b\|_{\widetilde{H}_{1}^{1}(X)} \leq C \alpha\left(\lambda\left(U_{+}\right)+\lambda\left(U_{-}\right)\right)$. Let

$$
\alpha_{ \pm}(t)=\left(\mathcal{M}_{\Omega_{ \pm}}\left(\left|f_{ \pm}\right|+\frac{\left|f_{ \pm}\right|}{r}+\left|\nabla f_{ \pm}\right|\right)\right)^{*}(t), \quad \alpha=\max \left(\alpha_{+}(t), \alpha_{-}(t)\right) .
$$

Remark that

$$
\begin{aligned}
\alpha_{+}(t) & \lesssim\left(\left|f_{+}\right|^{* *}+\left(\frac{\left|f_{+}\right|}{r}\right)^{* *}+\left|\nabla f_{+}\right|^{* *}\right)(t) \\
& \lesssim\left(|f|^{* *}+\left(\frac{|f|}{r}\right)^{* *}+|\nabla f|^{* *}\right)(t)
\end{aligned}
$$

where the implicit constant depends only on the doubling constant of $\Omega_{+}$. We used the fact that $\left\{x \in \Omega_{+} ;\left|f_{+}(x)\right|>\lambda\right\} \subset\{x \in \Omega ;|f(x)|>\lambda\}$, hence $f_{+}^{*}(t) \leq$ $f^{*}(t)$. Similarly, $\left(\frac{f_{+}}{r}\right)^{*}(t) \leq\left(\frac{f}{r}\right)^{*}(t)$ and $\left|\nabla f_{+}\right|^{*}(t) \leq|\nabla f|^{*}(t)$.

As $U_{+}$is contained in

$$
\left\{x \in \Omega_{+} ; \mathcal{M}_{\Omega_{+}}\left(\left|f_{+}\right|+\frac{\left|f_{+}\right|}{r}+\left|\nabla f_{+}\right|\right)(x)>\alpha_{+}(t)\right\}
$$

we have $\lambda\left(U_{+}\right) \leq t$. Similarly we get $\lambda\left(U_{-}\right) \leq t$. This yields

$$
\begin{aligned}
K\left(f, t, \widetilde{H}_{1}^{1}, \widetilde{H}_{\infty}^{1}\right) & \leq\|b\|_{\widetilde{H}_{1}^{1}}+t\|g\|_{\widetilde{H}_{\infty}^{1}} \\
& \leq C t\left(f^{* *}(t)+\left(\frac{|f|}{r}\right)^{* *}(t)+|\nabla f|^{* *}(t)\right) .
\end{aligned}
$$


For the general case when $f \in \widetilde{H}_{1}^{1}(X)+\widetilde{H}_{\infty}^{1}(X)$, we apply a similar argument to that of [8] to obtain the upper bound. We omit details.

Proof of Theorem 3.4. Set $\widetilde{H}_{p, 1}^{1}(X)=\left(\widetilde{H}_{1}^{1}(X), \widetilde{H}_{\infty}^{1}(X)\right)_{1-1 / p, p}$. By the reiteration theorem, it suffices to establish $\widetilde{H}_{p, 1}^{1}(X)=\widetilde{H}_{p}^{1}(X)$ with equivalent norms.

First, from the Calderón-Zygmund decomposition, we have $\widetilde{H}_{p}^{1}(X) \subset \widetilde{H}_{1}^{1}(X)+$ $\widetilde{H}_{\infty}^{1}(X)$ for $1<p<\infty$ where the inclusion is continuous.

From the previous results we have that for $f \in \widetilde{H}_{1}^{1}(X)+\widetilde{H}_{\infty}^{1}(X)$

$$
\begin{aligned}
\|f\|_{1-1 / p, p} & \sim\left\{\int_{0}^{\infty}\left(|f|^{* *}(t)+\left(\frac{|f|}{r}\right)^{* *}+|\nabla f|^{* *}(t)\right)^{p} d t\right\}^{1 / p} \\
& \sim\left\|f^{* *}\right\|_{p}+\left\|\left(\frac{|f|}{r}\right)^{* *}\right\|_{p}++\left\||\nabla f|^{* *}\right\|_{p} \\
& \sim\|f\|_{p}+\left\|\frac{f}{r}\right\|_{p}+\||\nabla f|\|_{p} \\
& \sim\|f\|_{\tilde{H}_{p}^{1}},
\end{aligned}
$$

where we used that for $l>1,\left\|f^{* *}\right\|_{l} \sim\|f\|_{l}$ (see [16], Chapter V, Lemma 3.21, p.191 and Theorem 3.21, p.201) .

\section{$5 \quad$ Restriction/Extension from/to $\mathbb{R}^{n}$}

We study the restriction operator onto $\Omega$. We construct an extension that is $p$ independent.

Theorem 5.1. Let $1 \leq p \leq \infty$.

- The restriction operator is bounded from $W_{p}^{1}\left(\mathbb{R}^{n}\right)$ into $H_{p}^{1}(X)$. Further, it is onto for $p \neq n$ and for $p=n$, its range is $\widehat{H}_{n}^{1}(X)$.

- There exists a linear extension operator $E$ that is bounded from $H_{p}^{1}(X)$ to $W_{p}^{1}\left(\mathbb{R}^{n}\right)$ if $p \neq n$ and from $\widehat{H}_{n}^{1}(X)$ to $W_{n}^{1}\left(\mathbb{R}^{n}\right)$.

This allows to recover the interpolation property of the $H_{p}^{1}(X)$ spaces in a quick fashion. Nonetheless, the direct proof in the previous sections could be of interest in other settings.

Observe also that this shows that $H_{n}^{1}(X)$ does not have the extension property.

We first study the restriction operator, then construct the extension and prove the ontoness together with the application to interpolation.

\subsection{Restriction}

The restriction operator $R$ is defined by $R(f)=\left.f\right|_{\Omega}$. Let $1 \leq p<\infty$. It is obvious that if $f \in W_{p}^{1}\left(\mathbb{R}^{n}\right)$ then $R(f) \in W_{p}^{1}(\Omega)$, and that $R: W_{p}^{1}\left(\mathbb{R}^{n}\right) \rightarrow$ $W_{p}^{1}(\Omega)$ is bounded. As $C_{0}^{\infty}\left(\mathbb{R}^{n}\right)$ is dense in $W_{p}^{1}\left(\mathbb{R}^{n}\right)$, the range is contained in $\widetilde{W}_{p}^{1}(\Omega)=H_{p}^{1}(X)$. 
For $p=n$, we show that $R$ maps into $\widehat{H}_{n}^{1}(X)$. Let $f \in W_{n}^{1}\left(\mathbb{R}^{n}\right)$ and let $g=R(f)$. Since we already know that $g \in H_{n}^{1}(X)$, it remains to show that $g_{a} / r \in L^{n}(X)$. Write $f=f_{\rho}+f_{\alpha}$ where $f_{\rho}(x)$ is here the average of $f$ on the whole sphere of radius $|x|$. Identifying $f_{\rho}$ with its restriction to $\Omega$, we see that $\left(f_{\rho}\right)_{r}=f_{\rho}$ and $\left(f_{\rho}\right)_{a}=0$. Thus, if we write $g=g_{r}+g_{a}$, we conclude that $g_{a}=\left(\left.f_{\alpha}\right|_{\Omega}\right)_{a}$, i.e $g_{a}(x)=f_{\alpha}(x)-f_{\Omega \cap S_{|x|}} f_{\alpha} d \sigma(\theta)$ for $x \in \Omega$. Thus

$$
\begin{aligned}
\int_{\Omega \cap S_{|x|}}\left|g_{a}\right|^{n} d \sigma(\theta) & \leq 2^{n} \int_{\Omega \cap S_{|x|}}\left|f_{\alpha}\right|^{n} d \sigma(\theta) \\
& \leq 2^{n} \int_{S_{|x|}}\left|f_{\alpha}\right|^{n} d \sigma(\theta) \\
& \leq C|x|^{n} \int_{S_{|x|}}\left|\nabla_{\theta} f_{\alpha}\right|^{n} d \sigma(\theta)
\end{aligned}
$$

where the last inequality is Poincare inequality $\left(P_{1}\right)$ on the sphere and $\nabla_{\theta}$ is the tangential gradient. Since $r(x)=|x|$ it follows that

$$
\begin{aligned}
\int_{\Omega}\left|\frac{g_{a}}{r}\right|^{n} d x & =\int_{0}^{+\infty} \int_{\Omega \cap S_{|x|}}\left|\frac{g_{a}}{r}\right|^{n} r^{n-1} d r d \sigma(\theta) \\
& \leq C \int_{0}^{+\infty} r^{n-1} \int_{S_{|x|}}\left|\nabla_{\theta} f_{\alpha}\right|^{n} d \sigma(\theta) d r \\
& \leq C \int_{\mathbb{R}^{n}}\left|\nabla f_{\alpha}\right|^{n} d x<\infty .
\end{aligned}
$$

Therefore $R(f) \in \widehat{H}_{n}^{1}(X)$.

For $p=\infty$, it is obvious that $R$ is bounded from $W_{\infty}^{1}\left(\mathbb{R}^{n}\right)$ into $H_{\infty}^{1}(X)$ (and it is onto by Whitney's extension theorem).

\subsection{Extension}

Let $1 \leq p \leq \infty$. Let $f \in H_{p}^{1}(X)$ if $p \neq n$ (resp. $f \in \widehat{H}_{n}^{1}(X)$ ). Write $f=f_{r}+f_{a}$ as in Section 3. Lemma 3.7 yields $f_{r} \in W_{p}^{1}\left(\mathbb{R}^{n}\right)$ and $f_{a} \in \widetilde{H}_{p}^{1}(X)$. It remains to extend $f_{a}$. We write $f_{a}=\left.f_{a}\right|_{\Omega_{+}}+\left.f_{a}\right|_{\Omega_{-}}=f_{a+}+f_{a-}$. We treat $f_{a+}$, the same analysis applying to $f_{a-}$. Let $\widetilde{\Omega}_{+}$be an open half-cone slightly larger than $\Omega_{+}$with same rotation axis. In spherical coordinates $(r, \theta, \phi)$ with $\theta \in[0, \pi]$, the angle to the positive $x_{n}$-axis, define $\psi_{+}(r, \theta, \phi)=(r, 2 w \theta / \pi, \phi)$ with $w$ the half-angle of $\Omega_{+}$. Then $\psi_{+}$is a bilipshitz map from $\mathbb{R}_{+}^{n}$ onto $\Omega_{+}$and from $\widetilde{\mathbb{R}^{n}}+$ onto $\widetilde{\Omega}_{+}$where $\widetilde{\mathbb{R}^{n}}$ is a slight extension of $\mathbb{R}_{+}^{n}$. We consider now the even extension $\zeta_{+}: W_{p}^{1}\left(\mathbb{R}_{+}^{n}\right) \rightarrow W_{p}^{1}\left(\mathbb{R}^{n}\right)$. Let $m_{+} \in C^{\infty}\left(\mathbb{R}^{n} \backslash\{0\}\right) \cap L^{\infty}\left(\mathbb{R}^{n}\right)$ such that $m_{+}(x)=g_{+}\left(\frac{x}{|x|}\right), m_{+}=1$ on $\mathbb{R}_{+}^{n}$ and $\operatorname{supp} m_{+} \subset \widetilde{\mathbb{R}^{n}} \cup\{0\}$. With these ingredients we define the extension $\xi_{+}\left(f_{a+}\right)$ of $f_{a+}$ as

$$
\xi_{+}\left(f_{a+}\right)=\left[m_{+} \zeta_{+}\left(f_{a+} \circ \psi_{+}\right)\right] \circ \psi_{+}^{-1} .
$$

It readily follows from the properties of $m_{+}$that

$$
\left\|m_{+} g / r\right\|_{p} \lesssim\|g / r\|_{p}
$$


and

$$
\left\|\left|\nabla\left(m_{+} g\right)\right|\right\|_{p} \lesssim\||\nabla g|\|_{p}+\|g / r\|_{p}
$$

for all $g \in W_{p}^{1}\left(\mathbb{R}^{n}\right)$. Using this fact, that bilipschitz maps preserve Sobolev spaces and density of Lipschitz functions, we obtain that $\xi_{+}\left(f_{a+}\right) \in W_{p}^{1}\left(\mathbb{R}^{n}\right)$ with $\operatorname{supp} \xi_{+}\left(f_{a+}\right) \subset \widetilde{\Omega}_{+}$and $\left\|\xi_{+}\left(f_{a+}\right)\right\|_{W_{p}^{1}\left(\mathbb{R}^{n}\right)} \leq C\left\|f_{a+}\right\|_{\widetilde{H}_{p}^{1}(X)}$. We conclude that $\xi\left(f_{a}\right)=\xi_{+}\left(f_{a+}\right)+\xi_{-}\left(f_{a-}\right) \in W_{p}^{1}\left(\mathbb{R}^{n}\right)$ is an extension of $f_{a}$ to $W_{p}^{1}\left(\mathbb{R}^{n}\right)$. Therefore, $E$ defined by

$$
E(f)=f_{r}+\xi\left(f_{a}\right)
$$

is an extension of $f$ to $W_{p}^{1}\left(\mathbb{R}^{n}\right)$. We have shown that the map $E$ is $H_{p}^{1}(X) \rightarrow$ $W_{p}^{1}\left(\mathbb{R}^{n}\right)$-bounded if $p \neq n$ and $\widehat{H}_{n}^{1}(X) \rightarrow W_{n}^{1}\left(\mathbb{R}^{n}\right)$-bounded if $p=n$.

\subsection{Relation to interpolation for $H_{p}^{1}(X)$}

From the previous subsections, we deduce that $R \circ E$ operates boundedly on $H_{p}^{1}(X)$ for $p \neq n$ and on $\widehat{H}_{n}^{1}(X)$ as the identity map. In particular, $R$ acting on $W_{p}^{1}\left(\mathbb{R}^{n}\right)$ is onto $H_{p}^{1}(X)$ for $p \neq n$ and onto $\widehat{H}_{n}^{1}(X)$ for $p=n$. Using the preservation of interpolation properties for retract diagrams, it follows that

$$
\left.\left(H_{1}^{1}(X), H_{\infty}^{1}(X)\right)_{1-1 / p, p}=R\left(W_{1}^{1}\left(\mathbb{R}^{n}\right), W_{\infty}^{1}\left(\mathbb{R}^{n}\right)\right)_{1-1 / p, p}\right)=R\left(W_{p}^{1}\left(\mathbb{R}^{n}\right)\right) .
$$

Therefore $\left(H_{1}^{1}(X), H_{\infty}^{1}(X)\right)_{1-1 / p, p}=H_{p}^{1}(X)$ for $p \neq n$ and $\widehat{H}_{n}^{1}(X)$ for $p=n$.

\section{Homogeneous versions}

Homogeneous Sobolev spaces are defined up to a constant, removing control on the $L^{p}$ norms on $f$. Since the vertex point plays a specific role, it is best here to fix the floatting constant by imposing control at this vertex point. We adopt the following definitions. Let $\operatorname{Lip}^{0}(X)$ be the space of Lipschitz functions in $X$ vanishing at 0 . For $1 \leq p \leq \infty$, we set

$$
\begin{gathered}
E_{p}=\left\{f \in \operatorname{Lip}^{0}(X) ;\||\nabla f|\|_{L^{p}(X)}<\infty\right\} \\
\widetilde{E}_{p}=\left\{f \in \operatorname{Lip}^{0}(X) ;\||\nabla f|\|_{L^{p}(X)}+\|f / r\|_{L^{p}(X)}<\infty\right\}
\end{gathered}
$$

Then $E_{p}$ and $\widetilde{E}_{p}$ are normed spaces and we call $\mathcal{H}_{p}^{1}(X)$ and $\widetilde{\mathcal{H}}_{p}^{1}(X)$ their completions. Clearly $\widetilde{E}_{\infty}=\widetilde{\mathcal{H}}_{\infty}^{1}(X)=E_{\infty}=\mathcal{H}_{\infty}^{1}(X)=\operatorname{Lip}^{0}(X)$.

It is easy to show that $\mathcal{H}_{p}^{1}(X)$ is composed of locally $p$-integrable functions. For $p>n$, one has (2.1) from the Morrey embedding and $f(0)=0$.

It is clear that $\widetilde{\mathcal{H}}_{p}^{1}(X) \subset \mathcal{H}_{p}^{1}(X)$ but for $1 \leq p \leq n$ the inclusion is strict 3 . Indeed a Lipschitz function supported away from 0 which agrees with $e^{i|x|^{-\alpha}}$ for $|x| \geq 1$ satisfies $\|f / r\|_{p}=\infty$ and belongs to $E_{p}$ if $\alpha>0$ is large enough. For $p>n$, the inclusion is an equality as we shall see.

Lemma 6.1. For $1 \leq p<\infty, \operatorname{Lip}^{0}(X) \cap \operatorname{Lip}_{0}(X)$ is dense in $\widetilde{\mathcal{H}}_{p}^{1}(X)$.

\footnotetext{
${ }^{3}$ In contrast with the inhomogeneous case for $p<n$.
} 
Proof. If $f \in \widetilde{E}_{p}$, consider $f_{k}=f \chi(r / k), k \in \mathbb{N}^{*}$, where $\chi:[0, \infty) \rightarrow[0,1]$ is a smooth function which is 1 on $[0,1]$ with support in $[0,2]$. It is easy to show that $\left\|\left|\nabla\left(f-f_{k}\right)\right|\right\|_{p}$ and $\left\|\left(f-f_{k}\right) / r\right\|_{p}$ tend to 0 as $k$ tends to $\infty$.

Remark. From there, one can see that the restrictions to $\Omega$ of functions in $C_{0}^{\infty}\left(\mathbb{R}^{n}\right)$ that vanish at 0 form a dense subspace of $\widetilde{\mathcal{H}}_{p}^{1}(X)$.

Corollary 6.2. $\quad$ For $1 \leq p<n$, (3.3) holds on $\widetilde{\mathcal{H}}_{p}^{1}(X)$.

- For $n<p<\infty$, (3.3) holds on $\mathcal{H}_{p}^{1}(X)$ and $\mathcal{H}_{p}^{1}(X)=\widetilde{\mathcal{H}}_{p}^{1}(X)$.

Proof. Assume first that $1 \leq p<n$. Then by the previous lemma, one can assume that $f \in \operatorname{Lip}_{0}(X)$ for which the argument of (3.3) applies.

Assume now $n<p<\infty$. Let $f \in \operatorname{Lip}^{0}(X)$. For $0<\epsilon<R<\infty$, set $A=\int_{\Omega_{+} \cap\{R>|x|>\epsilon\}}\left|\frac{f}{r}\right|^{p} d x$. Then argue as in the proof of (3.3). In the integration by parts, one picks an extra term which has a negative sign because $n-p<0$. Thus one can cancel it and obtain $A \leq C\|\| \nabla f \|_{L^{p}\left(X_{+}\right)}^{p}$ with $C$ independent of $\epsilon, R$. Taking limits and doing the same thing on $\Omega_{-}$shows that $f \in \widetilde{E}_{p}$ and we are done.

The first item also show that the closure in $\mathcal{H}_{p}^{1}(X)$ of $\operatorname{Lip}^{0}(X) \cap \operatorname{Lip}_{0}(X)$ is $\widetilde{\mathcal{H}}_{p}^{1}(X)$.

Theorem 6.3. The family $\left(\widetilde{\mathcal{H}}_{p}^{1}(X)\right)_{1 \leq p \leq \infty}$ is an interpolation family for the real method. Hence the same is true for $\left(\mathcal{H}_{p}^{1}(X)\right)_{n<p \leq \infty}$.

The proof for the spaces $\widetilde{\mathcal{H}}_{p}^{1}(X)$ is a minor adapatation of the one of Theorem 3.4 and is left to the reader. The second point follows from the above corollary.

Interpolation for the spaces $\mathcal{H}_{p}^{1}(X)$ for $p \leq n$ is unclear.

\section{Some remarks and generalizations}

Remark. (some explicit extensions) There are many extension operators. The following example was communicated to us by M. Pierre. For the (double) cone of $\mathbb{R}^{2}$ consisting of the 2 quadrants defined by $x y>0$, then one can take

$$
E f(x, y)= \begin{cases}f(x, y), & \text { if } x y>0 \\ \frac{x^{2} f(x,-y)+y^{2} f(-x, y)}{x^{2}+y^{2}}, & \text { if } x y<0 .\end{cases}
$$

Remark. (central role of the vertex) The analysis in this article does not use the fact that the cone $\Omega$ is symmetric under $x \mapsto-x$ and also does not use the specific opening angle. This means that the upper and lower (open) halfcones can be replaced by two half-cones located independently of one another provided they share the same vertex and that they are strictly separated by a hyperplane passing through the vertex and not containing any direction of the boundaries. Also the (finite) number of disjoint half-cones is not limited to 2 provided each pair satisfies the above requirements.

Remark. (other types of cones) The half-cones can be replaced by $\mathbb{R}_{+}^{*} \times N$ where $N$ is a Lipschitz domain on the unit sphere. On such domains, one has Poincaré inequalities with any exponents and this allows to adapt the arguments. 
Remark. (local geometry) Of course, the analysis done with inhomogeneous norms is stable by (smooth) truncation of the cone away from the vertex point. For example, if one wants to work on a truncated cone by requiring $r<1$, then one can use local variants as in N. Badr's thesis [2]. Details are left to the reader.

\section{References}

[1] R. Adams, Sobolev spaces. Pure and Applied Mathematics, Vol. 65. Academic Press, New York-London, 1975.

[2] N. Badr, Ph.D Thesis, Université Paris-Sud, 2007.

[3] N. Badr, Real interpolation of Sobolev Spaces, arXiv:0705.2216. To appear in Math. Scand.

[4] C. Bennett, R. Sharpley, Interpolation of operators, Academic Press, 1988.

[5] J. Bergh, J. Löfström, Interpolation spaces, An introduction, Springer (Berlin), 1976.

[6] R. Coifman, G. Weiss, Analyse harmonique sur certains espaces homogènes, Lecture notes in Math., Springer, 1971.

[7] M. Costabel, M. Dauge, S. Nicaise, Singularities of Maxwell interface problems. M2AN Math. Model. Numer. Anal. 33 (1999), no. 3,

[8] R. Devore, K. Scherer, Interpolation of linear operators on Sobolev spaces, Ann. of Math., 109, 583-599, 1979.

[9] P. Hajlasz, P. Koskela, Sobolev met Poincaré, Mem. Amer. Math. Soc., 2000, 145, (688), 1-101.

[10] P. Hajlasz, Sobolev spaces on a arbitrary metric space, Potential Anal., 1996, 5, 403-415.

[11] P. Hajlasz, P. Koskela, H. Tuominen, Sobolev embeddings, extensions and measure density condition, J. Funct. Anal., 2008, 254, 1217-1234.

[12] J. Heinonen, Lectures on analysis on metric spaces, Springer-Verlag, 2001.

[13] V. Maz'ya, Sobolev spaces. Springer-Verlag, Berlin, 1985. xix+486 pp.

[14] V. Maz'ya, S. Poborchi, Differentiable functions on bad domains. World Scientific Publishing Co., Inc., River Edge, NJ, 1997.

[15] E.M. Stein, Singular integrals and differentiability properties of functions. Princeton Mathematical Series, No. 30 Princeton University Press, Princeton, N.J. 1970.

[16] E. M. Stein, G. Weiss, Introduction to Fourier Analysis in Euclidean spaces, Princeton University Press, 1971.

[17] W. Ziemer, Weakly differentiable functions. Sobolev spaces and functions of bounded variation. Graduate Texts in Mathematics, 120. Springer-Verlag, New York, 1989. 